\title{
Variation of Superficial Palmar Arch a Case Report
}

\author{
Dr.D.Ashalatha; Head of the dept, Dr.Krupa Elena, Dr. Ravindra Kishore, \\ Dr. K.V.Ram Prasad, Dr. L.Lakshmi Sailaja. \\ Department of Anatomy, Andhra Medical College, Andhra Pradesh.
}

\begin{abstract}
The anastomoses between radial and ulnar arteries in the palm play a significant role in diseases of the palm through collateral circulation. During routine dissection of the upper limb of a 45-year-old male cadaver, we observed the superficial palmar arch (SPA) formed exclusively by the superficial branch of the ulnar artery. The superficial palmar branch of the radial artery entered the hand above the thenar muscles and provided palmar digital branches to the radial side of the index finger and the ulnar side of the thumb, without any contribution to the SPA. However, the radial side of the thumb was supplied by a branch from the deep palmar arch. The superficial branch of the ulnar artery gave origin to three common palmar digital arteries to supply the contiguous sides of the index, middle, ring and little fingers. It also provided origin to a digital branch to the ulnar side of the little finger.
\end{abstract}

Keywords: Radial artery, Superficial palmar arch, Ulnar artery

\section{Introduction}

The anastomoses between radial and ulnar arteries in the palm play a significant role in diseases of the palm through collateral circulation. During routine dissection of the MBBS students we have seen that the hand of a75 year-old male cadaver, showed variation in the completion of the superficial palmar arch (SPA) formed exclusively by the superficial branch of the ulnar artery. [The superficial arteries of the hand formed several diversified patterns that permitted into well-defined categories. About two-third of the SPA is formed by the ulnar artery alone; a further third is completed by the superficial palmar branch of the radial artery and a third either by the arteria radialis indicis or by the princeps pollicis or by the median artery. A classic type of SPA in which the superficial branch of the radial artery joins the superficial branch of the ulnar artery is found only in $34.5 \%$ of the cases. There are many reports regarding formation of SPA. In a study by Coleman et al., the complete arch was found in $78.5 \%$ of the cases and incomplete arch in the remaining $21.5 \%$, and this formed a major underlying factor in the aetiology of digital ischaemia. Ikeda et al. conducted stereoscopic arteriography of 220 cadaver hands and reported complete SPA in $96.4 \%$ of the cases, and only $3.6 \%$ had an incomplete arch. Gellman et al. showed a complete SPA in $84.4 \%$ and Al Turk and Metcalf reported complete SPA in $84 \%$ of the cases.

\section{Case Report}

The superficial palmar branch of the radial artery entered the hand through the thenar muscles at the flexor polices brevis and passed superficial to the thenar muscles and provided palmar digital branches to the radial side of the index finger and the ulnar side of the thumb, without any contribution to the SPA. However, the radial side of the thumb was supplied by a branch from the deep palmar arch. The superficial branch of the ulnar artery gave origin to three common palmar digital arteries to supply the contiguous sides of the index, middle, ring and little fingers. It also provided [Figure 1]. aproper palmar digital branch to the ulnar side of the little finger. Dissection of the left palm showing the superficial palmar arch formed solely by the superficial branch of the ulnar artery. Common palmar digital arteries; Proper palmar digital branches; SbU: superficial branch of the ulnar artery; SpR: Superficialpalmar branch of radial artery. On the right hand too the SPA is formed solely by the Superficial palmar branch of ulnar artery. [Figure: 2]. 


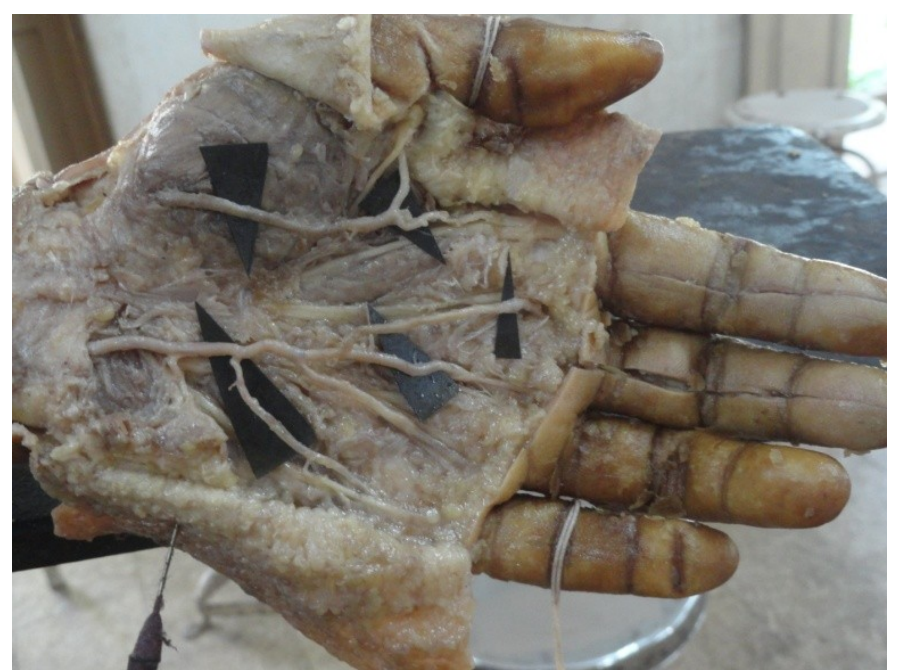

Figure 1:Dissection ofLeft hand of cadaver showing anomaly of superficial palmar arch.

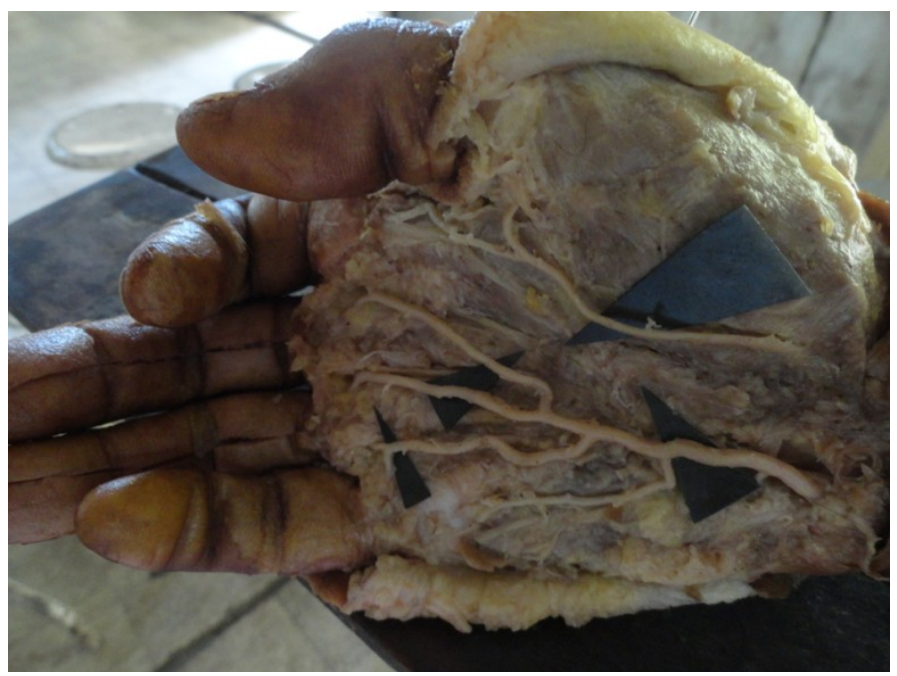

Figure 2: Dissection ofRight hand of cadaver showing anomaly of superficial palmar arch

\section{Discussion}

Knowledge of the anatomical variations of the arterial pattern of the hand is crucial for safe and successful hand surgery. The hand surgeon should keep in mind this kind of variation while performing surgical procedures such as, arterial repairs, vascular graft applications and free and/or pedicled flaps. While harvesting the radial artery for use as arterial by-pass conduits or while harvesting the free Radial Forearm Flap, the need to look specifically for variation in collateral circulation, like presence of incomplete SPA is a must. Currently, the methods of assessing hand circulation include the modified Allen test, Doppler ultrasonography and Photoplethysmography. Doppler study is a useful tool in pre-operative screening for radial artery harvesting for myocardial revascularisation.

SPA is an anastomosis, fed mainly by the ulnar artery. When the ulnar artery is occluded, the viability of the structures in the palm supplied by the ulnar artery depends on the efficacy of the collateral circulation. In the present case, there was no anastomosis between the ulnar artery and the radial or median or interosseous arteries. Thus, in ulnar artery occlusion in cases like ours, there will be no collateral flow of blood to meet the metabolic demands of the palmar tissue, and this will result in acute ischaemia, manifested by Claudications, rest pain and/or gangrene.

\section{References:}

[1]. Willams PL, Bannister LG, Berry MM. Gray's Anatomy. 38th ed. New York: Churchill Livingstone; 2000. p. 1544.

[2]. Moore KL, Dalley AF. Clinically Oriented Anatomy. 10th ed. Philadelphia: Lippincott, Williams and Wilkins; 1999. p. 751.

[3]. Coleman SS, Anson BJ. Arterial patterns in the hand based upon a study of 650 specimens. Surg Gynecol Obstet. 1961; 113:40924.

[4]. Ikeda A, Ugawa A, Kazihara Y, Hamada N. Arterial patterns in the hand based on a three-dimensional analysis of 220 cadaver hands. J Hand Surg Am. 1988;13:501-9. 
[5]. Gellman H, Botte MJ, Shankwiler J, Gelberman RH. Arterial patterns of the deep and superficial palmar arches. Clin Orthop Relat Res. 2001;383:41-6.

[6]. Al-Turk M, Metcalf WK. A study of the superficial palmar arteries using the Doppler Ultrasonic Flowmeter. J Anat. 1984;138:2732.

[7]. Ruengsakulrach P, Brooks M, Hare DL, Gordon I, Buxton BF. Preoperative assessment of hand circulation by means of Doppler ultrasonography and the modified Allen test. J Thorac Cardiovasc Surg.2001;121:526-31.

[8]. Pola P, Serricchio M, Flore R, Manasse E, Favuzzi A, Possati GF. Safe removal of the radial artery for myocardial revascularization: A Doppler study to prevent ischemic complications to the hand. J Thorac Cardiovasc Surg. 1996;112:737-44. 